\title{
Short communication: Calculating analytical reliabilities for single-step predictions
}

\section{Edel, ${ }^{*}$ E. C. G. Pimentel, M. Erbe, R. Emmerling, and K.-U. Götz}

Institute of Animal Breeding, Bavarian State Research Center for Agriculture, 85586 Grub, Germany

\section{ABSTRACT}

It has been shown that single-step genomic BLUP (ssGBLUP) can be reformulated, resulting in an equivalent SNP model that includes the explicit imputation of gene contents of all ungenotyped animals in the pedigree. This reformulation reveals the underlying mechanism enabling ungenotyped animals to contribute information to genotyped animals via estimates of marker effects and consequently to the reliability of genomic predictions, a key feature generally associated with the single-step approach. Irrespective of which BLUP formulation is used for genomic prediction, with increasing numbers of genotyped animals, the markeroriented model is recommended when calculating the reliabilities of genomic predictions. This approach has the advantage of a manageable and stable size of the model matrix that needs to be inverted to calculate analytical prediction error variances of marker effects, an advantage that also holds for prediction with the single-step model. However, when including imputed genotypes in the design matrix of marker effects, an additional imputation residual term has to be considered to account for the prediction error of imputation. We summarize some of the theoretical aspects associated with the calculation of analytical reliabilities of singlestep predictions. Derivations are based on the equivalent reformulation of ssGBLUP as a marker-oriented model and the calculation of prediction error variances of marker effects. We propose 2 approximations that allow for a substantial reduction of the complexity of the matrix operations involved, while retaining most of the relevant information required for reliability calculations. We additionally provide a general framework for an implementation of single-step reliability approximation using standard animal model reliabilities as a starting point. Finally, we demonstrate the effectiveness of the proposed approach using a small example extracted

Received September 17, 2018

Accepted December 15, 2018.

${ }^{*}$ Corresponding author: Christian.Edel@LfL.bayern.de from data of the routine evaluation on dual-purpose Fleckvieh (Simmental) cattle.

Key words: genomic breeding value, genotype imputation, reliability approximation, single-step genomic BLUP (ssGBLUP)

\section{Short Communication}

In single-step genomic BLUP (ssGBLUP), information of genotyped (gt) and nongenotyped (ngt) animals is combined in a single, large estimation model. In a standard formulation that conceptually corresponds to the standard genomic BLUP (GBLUP) model, the mixed model equations (MME) of the single-step model are (Aguilar et al., 2010)

$$
\left[\begin{array}{ccc}
\mathbf{X}^{\prime} \mathbf{R}^{-1} \mathbf{X} & \mathbf{X}^{\prime} \mathbf{R}^{-1} \mathbf{Z} \\
\mathbf{Z}^{\prime} \mathbf{R}^{-1} \mathbf{X} & \mathbf{Z}^{\prime} \mathbf{R}^{-1} \mathbf{Z}+\mathbf{H}^{-1} & \frac{1}{\sigma_{a}^{2}}
\end{array}\right]\left[\begin{array}{l}
\hat{\mathbf{b}} \\
\hat{\mathbf{a}}
\end{array}\right]=\left[\begin{array}{l}
\mathbf{X}^{\prime} \mathbf{R}^{-1} \mathbf{y} \\
\mathbf{Z}^{\prime} \mathbf{R}^{-1} \mathbf{y}
\end{array}\right],
$$

where $\mathbf{H}$ is a combined relationship matrix of gt and ngt animals of the form

$$
\mathbf{H}=\left[\begin{array}{cc}
\mathbf{A}_{11}-\mathbf{A}_{12} \mathbf{A}_{22}^{-1} \mathbf{A}_{21}+\mathbf{A}_{12} \mathbf{A}_{22}^{-1} \mathbf{G A}_{22}^{-1} \mathbf{A}_{21} & \mathbf{A}_{12} \mathbf{A}_{22}^{-1} \mathbf{G} \\
\mathbf{G A}_{22}^{-1} \mathbf{A}_{21} & \mathbf{G}
\end{array}\right],
$$

where subscripts 1 and 2 refer to ngt and gt animals, respectively; $\mathbf{G}$ is a genomic relationship matrix; $\mathbf{X}$ and $\mathbf{Z}$ are design matrices for fixed and random effects, respectively; $\mathbf{R}$ is the residual covariance matrix; $\mathbf{y}$ is the vector of observations; and $\mathbf{A}$ is the numerator relationship matrix. Christensen and Lund (2010) noted that matrix $\mathbf{H}$ can be conceptually divided into 2 matrices:

$$
\begin{aligned}
\mathbf{H} & =\mathbf{H}_{r e s}+\mathbf{H}_{i m p}=\left[\begin{array}{cc}
\mathbf{A}_{11}-\mathbf{A}_{12} \mathbf{A}_{22}^{-1} \mathbf{A}_{21} & 0 \\
0 & 0
\end{array}\right] \\
& +\left[\begin{array}{cc}
\mathbf{A}_{12} \mathbf{A}_{22}^{-1} \mathbf{G A}_{22}^{-1} \mathbf{A}_{21} & \mathbf{A}_{12} \mathbf{A}_{22}^{-1} \mathbf{G} \\
\mathbf{G A}_{22}^{-1} \mathbf{A}_{21} & \mathbf{G}
\end{array}\right],
\end{aligned}
$$


where $\mathbf{H}_{i m p}$ is a relationship matrix based on observed and imputed genotypes, and $\mathbf{H}_{\text {res }}$ is the covariance matrix of the error of (implicit) imputation. The term "imputation" is used here to describe the process of estimating unobserved gene contents $(\widehat{\text { gc }})$ by inference on observed gene contents (Gengler et al., 2007):

$$
\widehat{\mathbf{g c}}_{1}=\mathbf{A}_{12} \mathbf{A}_{22}^{-1} \mathbf{g c}_{2}
$$

$\mathbf{H}_{\text {res }}$ therefore describes a component that cannot be covered by the imputed genotypes alone because of their properties as estimates and can be interpreted as the error of imputation.

With this structural separation, it becomes apparent that the information contributed by ngt animals to gt animals is completely transmitted through the imputed genotype, whereas, for ngt animals, an additional informational component has to be considered (termed the "imputation residual"). Using this formulation, Fernando et al. (2014) proposed a model that is equivalent to ssGBLUP similar to the equivalence between the SNP-BLUP and GBLUP models. The MME for this equivalent SNP-based single-step model are

$$
\left[\begin{array}{ccc}
\mathbf{X}^{\prime} \mathbf{R}^{-1} \mathbf{X} & \mathbf{X}^{\prime} \mathbf{R}^{-1} \mathbf{M} & \mathbf{X}_{1}^{\prime} \mathbf{R}_{1}^{-1} \mathbf{Z}_{1} \\
\mathbf{M}^{\prime} \mathbf{R}^{-1} \mathbf{X} & \mathbf{M}^{\prime} \mathbf{R}^{-1} \mathbf{M}+\mathbf{I} \frac{1}{\sigma_{g}^{2}} & \mathbf{M}_{1}^{\prime} \mathbf{R}_{1}^{-1} \mathbf{Z}_{1} \\
\mathbf{Z}_{1}^{\prime} \mathbf{R}_{1}^{-1} \mathbf{X}_{1} & \mathbf{Z}_{1}^{\prime} \mathbf{R}_{1}^{-1} \mathbf{M}_{1} & \mathbf{Z}_{1}^{\prime} \mathbf{R}_{1}^{-1} \mathbf{Z}_{1}+\mathbf{A}^{11} \frac{1}{\sigma_{a}^{2}}
\end{array}\right]\left[\begin{array}{c}
\hat{\mathbf{b}} \\
\hat{\mathbf{g}} \\
\hat{\varepsilon}
\end{array}\right]=\left[\begin{array}{c}
\mathbf{X}^{\prime} \mathbf{R}^{-1} \mathbf{y} \\
\mathbf{M}^{\prime} \mathbf{R}^{-1} \mathbf{y} \\
\mathbf{Z}_{1}^{\prime} \mathbf{R}_{1}^{-1} \mathbf{y}_{1}
\end{array}\right],
$$

where $\mathbf{M}$ refers here to a matrix of imputed and observed genotypes of all animals in the pedigree; $\mathbf{M}_{1}$ is a submatrix of imputed genotypes of ngt animals with phenotype; $\mathbf{Z}_{1}$ is a design matrix relating phenotypes of ngt animals to all ngt animals; $\mathbf{I}$ is the identity matrix; and vectors $\mathbf{b}, \mathbf{g}$, and $\varepsilon$ refer to estimates for fixed effects and random marker and imputation residual effects, respectively. Parameter $\sigma_{g}^{2}=\sigma_{a}^{2} / \sum_{j=1}^{m}\left(2 p_{j} q_{j}\right)$, where $\sigma_{a}^{2}$ is the additive genetic variance of the trait, $p_{j}$ and $q_{j}$ are estimates of base allele frequencies, and $m$ is the number of markers used in the analysis. $\mathbf{A}^{11}$ is developed using a standard formula for partitioned matrix inversion and is the sub-block of the inverse of the numerator relationship matrix based on the full pedigree that corresponds to ngt animals. This means that $\mathbf{A}^{11}=\left(\mathbf{A}_{11}-\mathbf{A}_{12} \mathbf{A}_{22}^{-1} \mathbf{A}_{21}\right)^{-1}$ is the inverse of the covariance of the imputation residual.

Direct inversion of the left-hand-side of the full MME presented above to derive the prediction error variance
(PEV) for SNP effects and imputation residuals might be an option if the number of genotyped animals were larger than the number of SNP used in the analysis. However, in most practical implementations, system size will be too large for direct inversion. We therefore propose to calculate PEV for marker effects from the equation above and to use them as a first step in the approximation of reliabilities of genomic breeding values (GBV) of all animals in the single-step system. However, when using observed and imputed genotypes to derive reliabilities for GBV based on the PEV of marker effects, it is not sufficient to simply invert the submatrix of marker effects $\left(\mathbf{M}^{\prime} \mathbf{R}^{-1} \mathbf{M}+\mathbf{I} \frac{1}{\sigma_{g}^{2}}\right)$, because the contribution of the imputation residual is not appropriately taken into account. The complete expression for the sub-block of the inverse of the left-hand-side can be deduced using standard formulas for partitioned matrix inversion assuming the following decomposition:

$$
\begin{aligned}
\mathbf{B} & =\left[\begin{array}{lll}
\mathbf{B}_{11} & \mathbf{B}_{12} & \mathbf{B}_{13} \\
\mathbf{B}_{21} & \mathbf{B}_{22} & \mathbf{B}_{23} \\
\mathbf{B}_{31} & \mathbf{B}_{32} & \mathbf{B}_{33}
\end{array}\right] \\
& =\left[\begin{array}{ccc}
\mathbf{X}^{\prime} \mathbf{R}^{-1} \mathbf{X} & \mathbf{X}^{\prime} \mathbf{R}^{-1} \mathbf{M} & \mathbf{X}_{1}^{\prime} \mathbf{R}_{1}^{-1} \mathbf{Z}_{1} \\
\mathbf{M}^{\prime} \mathbf{R}^{-1} \mathbf{X} & \mathbf{M}^{\prime} \mathbf{R}^{-1} \mathbf{M}+\mathbf{I} \frac{1}{\sigma_{g}^{2}} & \mathbf{M}_{1}^{\prime} \mathbf{R}_{1}^{-1} \mathbf{Z}_{1} \\
\mathbf{Z}_{1}^{\prime} \mathbf{R}_{1}^{-1} \mathbf{X}_{1} & \mathbf{Z}_{1}^{\prime} \mathbf{R}_{1}^{-1} \mathbf{M}_{1} & \mathbf{Z}_{1}^{\prime} \mathbf{R}_{1}^{-1} \mathbf{Z}_{1}+\mathbf{A}^{11} \frac{1}{\sigma_{a}^{2}}
\end{array}\right] .
\end{aligned}
$$

When ignoring the "11" block of fixed effects for the sake of clarity of notation, the " 22 " sub-block of the inverse of the left-hand-side corresponding to marker effects is

$$
\begin{aligned}
& \mathbf{B}^{22}=\left(\mathbf{B}_{22}-\mathbf{B}_{23} \mathbf{B}_{33}^{-1} \mathbf{B}_{32}\right)^{-1} \\
& =\left[\left(\mathbf{M}^{\prime} \mathbf{R}^{-1} \mathbf{M}+\mathbf{I} \frac{1}{\sigma_{g}^{2}}\right)\right. \\
& \left.-\mathbf{M}_{1}^{\prime} \mathbf{R}_{1}^{-1} \mathbf{Z}_{1}\left(\mathbf{Z}_{1}^{\prime} \mathbf{R}_{1}^{-1} \mathbf{Z}_{1}+\mathbf{A}^{11} \frac{1}{\sigma_{a}^{2}}\right)^{-1} \mathbf{Z}_{1}^{\prime} \mathbf{R}_{1}^{-1} \mathbf{M}\right]^{-1} .
\end{aligned}
$$

Calculating this expression would still be demanding, especially with terms like $\left(\mathbf{Z}_{1}^{\prime} \mathbf{R}_{1}^{-1} \mathbf{Z}_{1}+\mathbf{A}^{11} \frac{1}{\sigma_{a}^{2}}\right)^{-1}$ of dimension ngt $\times$ ngt being involved. However, once de- 
rived, the resulting PEV of SNP estimates will provide the basis of all information relevant for the calculation of the reliability of GBV of gt animals. Furthermore, there are easier approaches to derive the additional information provided by gt animals to ngt animals than explicitly calculating expressions analogous to $\mathbf{B}^{22}$ for all relevant sub-blocks.

Matrix $\mathbf{A}_{11}-\mathbf{A}_{12} \mathbf{A}_{22}^{-1} \mathbf{A}_{21}$ describes the covariances between ngt animals adjusted for the part of the covariance that can be derived from the block of gt animals and the relationships between gt and ngt animals. It can be interpreted as the PEV of imputation, where $\mathbf{A}_{11}$ represents the true pedigree relationship among ngt animals, and $\mathbf{A}_{12} \mathbf{A}_{22}^{-1} \mathbf{A}_{21}$ represents the variance of errors when imputing relationships from gt relatives. Examinations of some practical examples, albeit limited to the situation found in dairy cattle (e.g., large genotyped offspring groups on the male side, half-sib structure), suggest that with increasing numbers of gt animals, matrix $\mathbf{A}_{11}-\mathbf{A}_{12} \mathbf{A}_{22}^{-1} \mathbf{A}_{21}$ (and therefore $\mathbf{A}^{11}$ as well) tends to become diagonally dominant and might be approximated by a diagonal matrix. Depending on the design, this would lead to expression $\mathbf{Z}_{1}^{\prime} \mathbf{R}_{1}^{-1} \mathbf{Z}_{1}+\mathbf{A}^{11} \frac{1}{\sigma_{a}^{2}}$ being diagonal and easily invertible. We therefore propose here to use a diagonal matrix based on the vector $\mathbf{a}_{11}^{*}$ as an approximation, where $\mathbf{a}_{11}^{*}=\operatorname{diag}\left(\mathbf{A}_{11}\right) \times\left(1-\mathbf{R}_{i m p}^{2}\right)$, with $\mathbf{R}_{i m p}^{2}$ being the vector of individual reliabilities of imputation. The diagonal matrix approximation is finally calculated as $\mathbf{A}_{11}-\mathbf{A}_{12} \mathbf{A}_{22}^{-1} \mathbf{A}_{21} \approx \mathbf{D}$, where $\mathbf{D}$ is a diagonal matrix with diagonal element $d_{i}$ equal to the $i$ th element of vector $\mathbf{a}_{11}^{*}$. Individual reliabilities of imputation can be derived directly by dividing the diagonal of $\mathbf{A}_{12} \mathbf{A}_{22}^{-1} \mathbf{A}_{21}$ by the diagonal of $\mathbf{A}_{11}$, or more conveniently, by using standard software to calculate approximate reliabilities using the full pedigree and assuming a heritability of (close to) 1 and that only gt animals have an observation.

In single-step GBLUP, imputation serves 2 main purposes that do not necessarily coincide: (1) it establishes the mechanism that allows the flow of pedigree information from ngt animals to gt animals, and (2) it enlarges the reference group for SNP effect estimation with ngt animals whose genotypes can be imputed with high accuracy. The importance of (2) might not be initially obvious and might well depend on the structure of the population. Nevertheless, we have investigated this aspect in a previous study and found a considerable effect for small to medium-size genomic systems (Shabalina et al., 2017). An additional way to reduce the problem imposed by the dimensionality of
$\mathbf{Z}_{1}^{\prime} \mathbf{R}_{1}^{-1} \mathbf{Z}_{1}+\mathbf{A}^{11} \frac{1}{\sigma_{a}^{2}}$ is therefore to reduce the ngt animals considered in reliability calculations to those that contribute a relevant amount of information to the reliability of SNP estimation. A reduction of ngt animals would then have the additional benefit of reducing the expense of explicitly imputing and storing genotypes of all ngt animals; $\mathbf{R}_{i m p}^{2}$ seems to be a useful criterion to identify those ngt animals. However, for the results presented here, we used a more parsimonious approach and selected the subset of ngt animals for inclusion based on the following criterion: an ngt animal to be included is a sire, dam, or paternal or maternal grandsire of a gt animal. Applying this criterion leads to a strong reduction of dimensionality and a straightforward preparation of the necessary pedigree. Consequently, matrices involved in calculating the reliability (including $\mathbf{M}$ ) will be reduced to the dimensionality of gt animals plus the ngt animals defined as relevant.

Following the argument that the reliability of GBV $\left(\mathbf{R}_{g b v}^{2}\right)$ of gt animals is only determined by the resulting PEV of SNP and the 2 approximations presented above allows us to calculate these reliabilities as a very close approximation. This means the vector of $\mathbf{R}_{g b v}^{2}$ does represent final single-step reliabilities for gt animals. For the ngt animals, we did not calculate the final reliability explicitly but developed a simplified approach that is easier to implement in practical applications. The process starts by using $\mathbf{R}_{g b v}^{2}$ of gt animals and calculating the difference between these reliabilities and the conventional animal model reliabilities $\left(\mathbf{R}_{\text {conv }}^{2}\right)$ of gt animals by the approximate formula of Harris and Johnson (2010):

$$
\mathbf{R}_{p l u s}^{2}=\frac{\mathbf{R}_{g b v}^{2}-\mathbf{R}_{c o n v}^{2}}{\mathbf{R}_{g b v}^{2} \mathbf{R}_{c o n v}^{2}+1-2 \mathbf{R}_{c o n v}^{2}} ;
$$

$\mathbf{R}_{\text {plus }}^{2}$ of gt animals is then transformed into an effective observation equivalent (EOP) using formula

$$
\mathrm{EOP}_{\text {plus }}=\lambda \frac{\mathbf{R}_{\text {plus }}^{2}}{1-\mathbf{R}_{\text {plus }}^{2}},
$$

with $\lambda$ being $\left(\sigma_{e}^{2} / \sigma_{a}^{2}\right)$, the ratio of the residual variance $\left(\sigma_{e}^{2}\right)$ to additive genetic variance for the trait under consideration. In the next step, the $\mathrm{EOP}_{\text {plus }}$ of gt animals are added to the "conventional" weight used for phenotypic observations in the analysis. For gt animals without own performance or offspring phenotypic infor- 
mation, this will introduce a nonzero weight. For gt animals with own or offspring phenotypic information, this will increase the weight of the observation. Then, using these weights and the parameter values of the trait, we calculated conventional reliabilities. Finally, we collected the resulting reliabilities from this calculation step for ngt animals and the already established $\mathbf{R}_{g b v}^{2}$ of gt animals directly into a single vector of approximated reliabilities for all animals in the single-step model $\left(\mathbf{R}_{\text {total }}^{2}\right)$.

For a better understanding of how the proposed method can be used in a practical application, an overview of the procedure is presented in Figure 1 as a workflow diagram. In the following, we comment on the steps of the procedure given in this diagram in more detail, providing a general framework for implementation.

Step 1. Reduce the pedigree to genotyped animals and all their ancestors back to the genetic base of the underlying full model. It has been shown that the information provided by nongenotyped daughters of a genotyped sire can be absorbed into the daughter yield deviations of the genotyped sire (Meuwissen et al., 2011) if these animals have no direct links to other genotyped animals. This step reduces the size of the problem considerably. The price to pay for this reduction is that the reliability gain provided by genomic information of the genotyped sire is not automatically propagated to the absorbed daughters, at least not when updated weights are used in the context of the reduced model only (see below). However, this propagation can also be implemented as a post-processing operation. Proposals for this post-processing have already been made (see Harris and Johnson, 2010).

Step 2. Use a standard program to calculate $\mathbf{R}_{\text {conv }}^{2}$ for all animals in the evaluation. We assume this program to give approximately correct reliabilities even for complex models that include several fixed or random effects.

Step 3. Using the reduced pedigree from step 1, deregress each animal's $\mathbf{R}_{\text {conv }}^{2}$ for the contribution of other members of the reduced pedigree. A detailed system-

1) Reduced pedigree of all gt animals and ancestors back to genetic base

2) Conventional $\mathbf{R}^{2}$ for all animals (young animals included)

3) Deregressed $\mathbf{R}^{2}$ for reduced pedigree -> derived weights

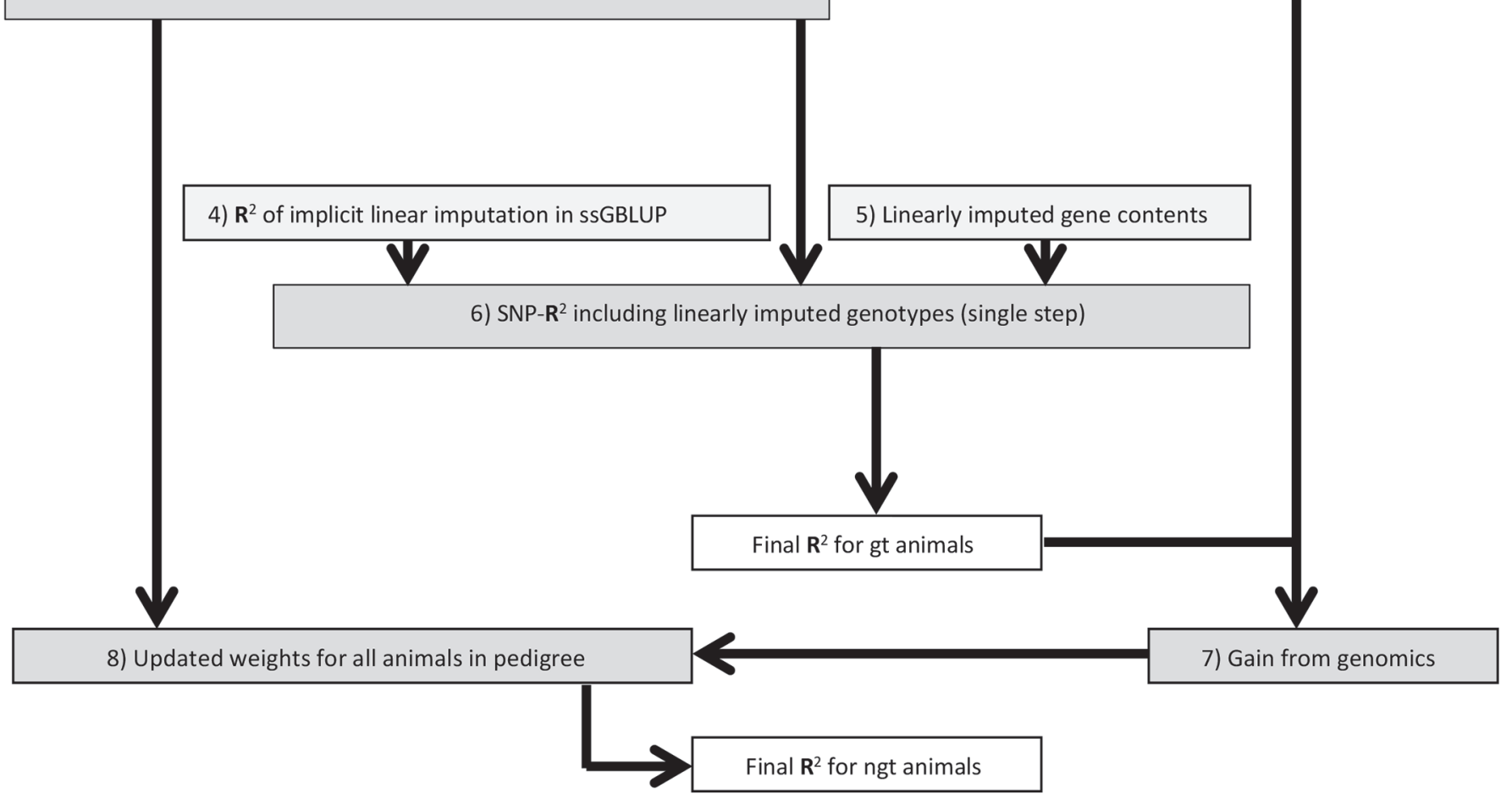

Figure 1. Proposed workflow for reliability calculation. gt = genotypes; ngt $=$ nongenotyped; ssGBLUP $=$ single-step genomic BLUP; $\mathbf{R}^{2}$ $=$ reliability. 
atic description of the procedure proposed for deregressing reliabilities is given in Appendix A of Harris and Johnson (2010). Transform deregressed $\mathbf{R}^{2}$ into EOP to be used as weights in further calculation steps.

Step 4. Approximate $\mathbf{R}_{i m p}^{2}$ for all members of the reduced pedigree using standard software; for example, using dummy phenotypes for gt animals only and a heritability of (close to) 1 . This calculation step has to be done only once for all traits, given that the population used for predicting breeding values is constant across traits. Use these $\mathbf{R}_{i m p}^{2}$ for the diagonal approximation or selection of relevant contributors as described.

Step 5. Calculate and store imputed genotypes for all ngt animals in the reduced pedigree. For this pur-

a)

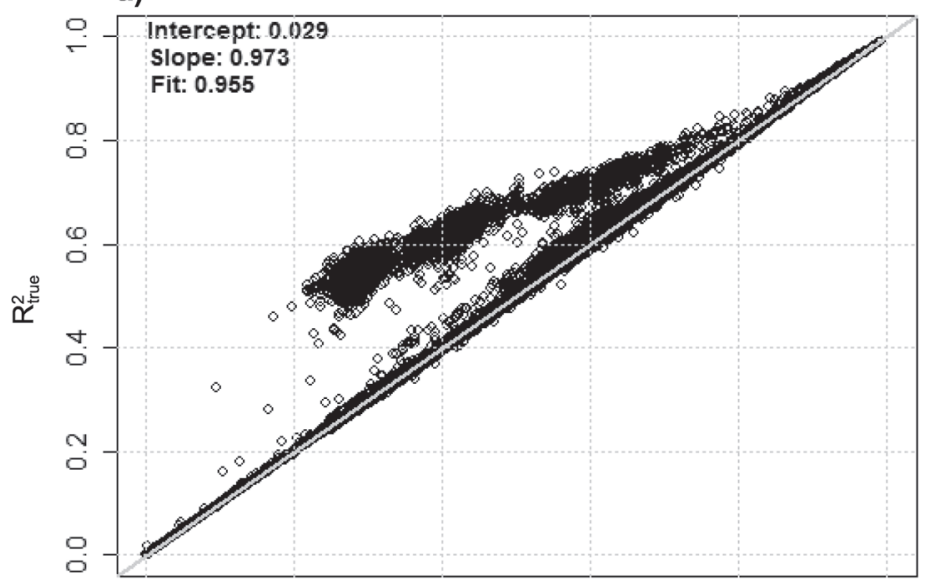

c)

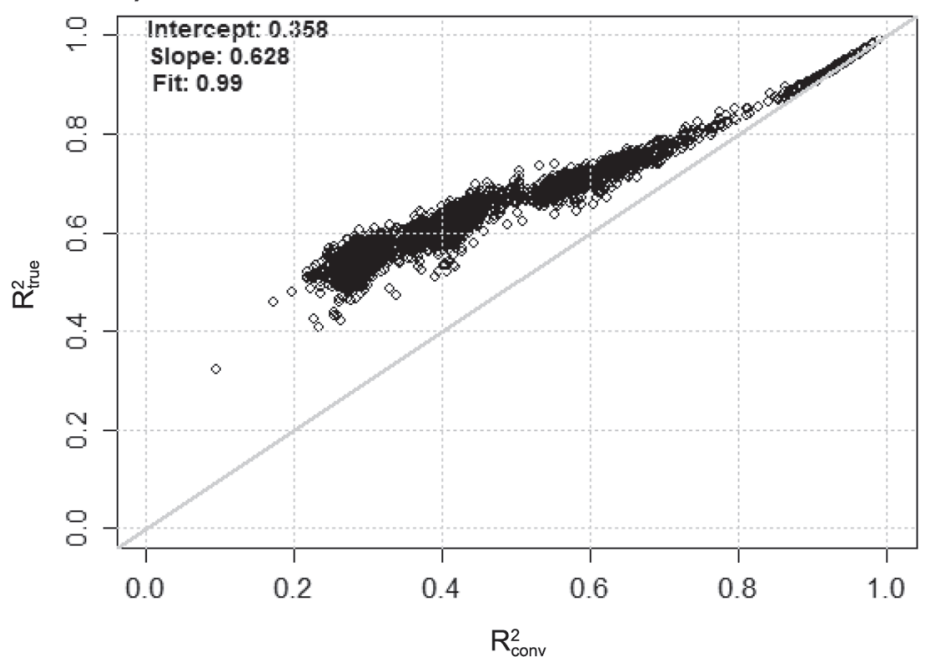

pose, Fernando et al. (2014) provided an extremely efficient algorithm based on sparse matrix operations that can easily be parallelized. This algorithm allows for the imputation of the genotypes of hundreds of thousands of animals within a few hours. Computational effort can be reduced drastically when considering relevant contributors only. This calculation step has to be done only once for all traits, given that the population used for predicting breeding values is constant across traits.

Step 6. Calculate $\mathbf{R}_{g b v}^{2}$ for gt animals via the PEV matrix of marker effects using observed and imputed genotypes. Apply the method and approximations as proposed in this study. Consider a global intercept in the calculation. This step directly produces $\mathbf{R}_{\text {total }}^{2}$ for gt animals. b)

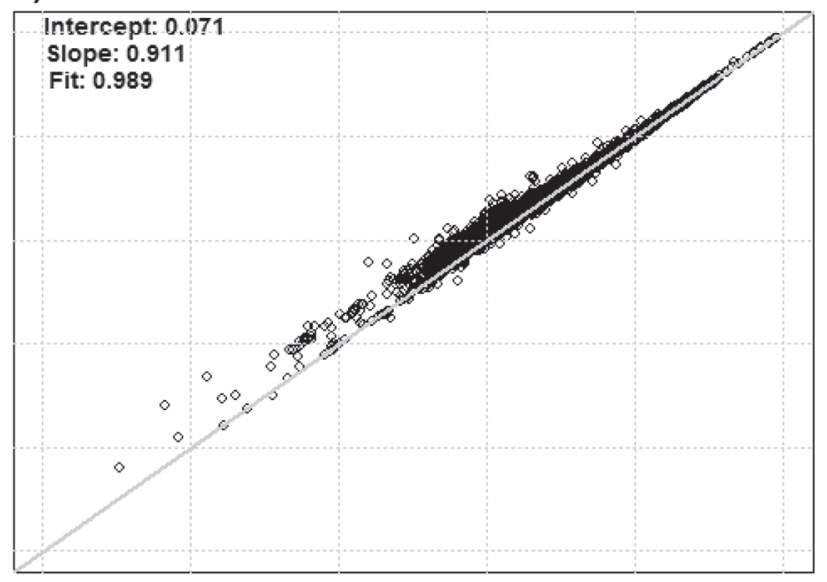

d)



Figure 2. Analytical reliability for single-step $\left(\mathbf{R}_{\text {true }}^{2}\right)$ derived by direct inversion versus analytical reliability of a conventional animal model derived by direct inversion $\left(\mathbf{R}_{\text {conv }}^{2}\right)$. Subplots are for (a) all animals in the pedigree, (b) nongenotyped (ngt) parents of genotyped (gt) animals only, (c) gt animals only, and (d) gt animals without own or offspring performance. 
Step \%. Calculate the gain from genomics for gt animals. This can be achieved by transforming $\mathbf{R}_{\text {conv }}^{2}$ and $\mathbf{R}_{g b v}^{2}$ of gt animals into EOP, as outlined above.

Step 8. Using updated weights as outlined calculate $\mathbf{R}_{\text {total }}^{2}$ for ngt animals using standard software for reliability approximation.

To demonstrate the efficiency of the proposed approximations, we applied the method to a test data set using real data from the Fleckvieh population. We extracted a test pedigree of 60,769 animals built from 6,701 genotyped animals, of which 3,045 also had a phenotype (EOP $>0$ ). From the 54,068 ngt animals included, 36,202 also had a phenotype. Phenotypes, $\mathbf{R}_{\text {conv }}^{2}$, and EOP were calculated for the trait milk yield

a)

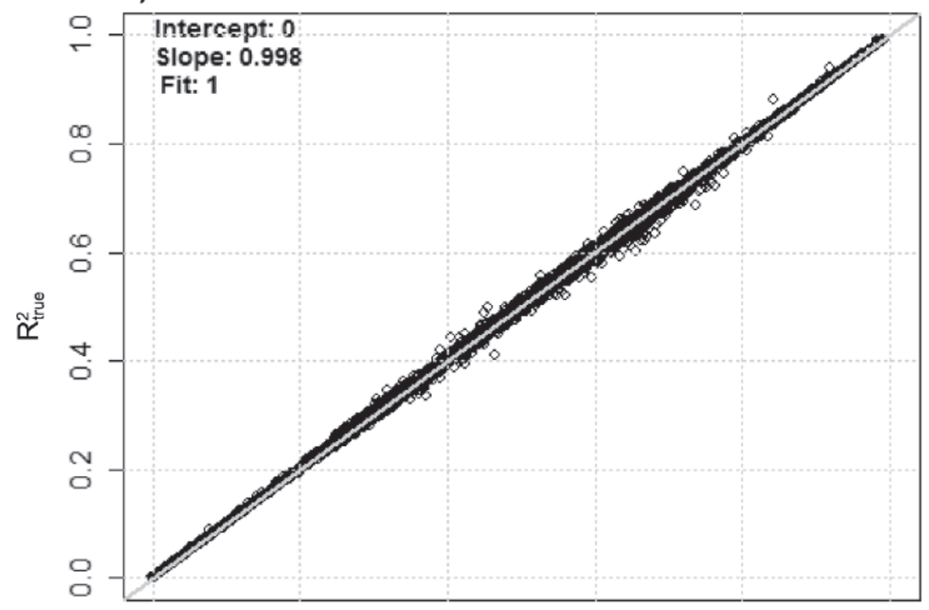

c)

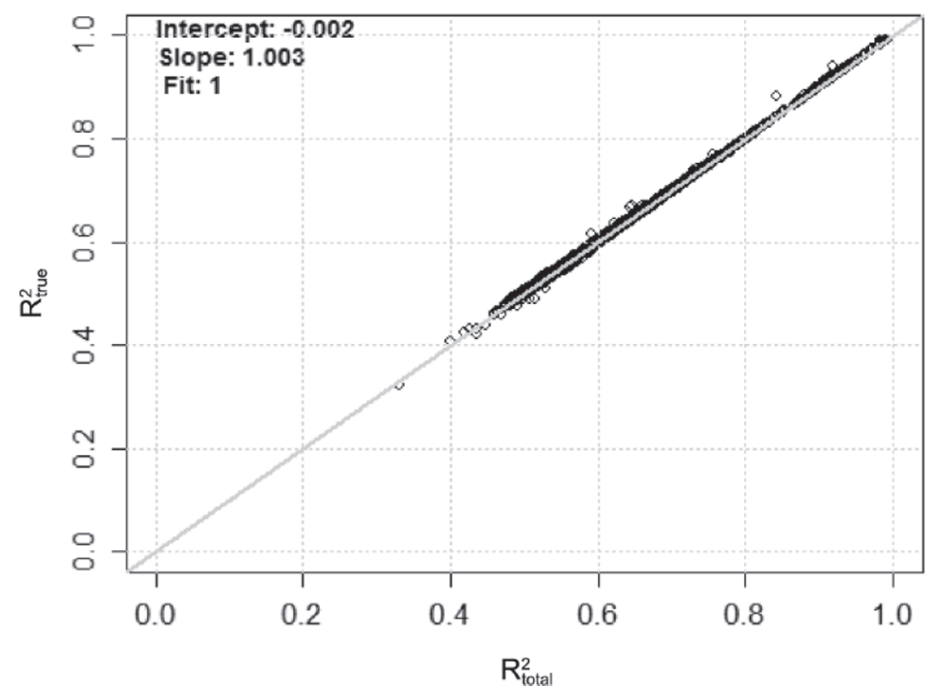

with heritability of 0.44 . Deregressed reliabilities and imputed genotypes were calculated as described above. Observed genotypes used for imputation were scaled by subtracting 2 times the base allele frequency from each of 41,040 marker columns (coded as gene content of the reference allele). In a first step, we set up the standard ssGBLUP model and inverted the left-hand-side directly to derive PEV and exact values for the singlestep reliabilities, which were used as the reference in the comparison of results $\left(\mathbf{R}_{\text {true }}^{2}\right)$. We then calculated approximate reliabilities for SNP and related reliabilities of GBV for gt animals using a combination of both approximations described above. Considering only relevant contributors resulted in a pedigree size of 13,396

b)

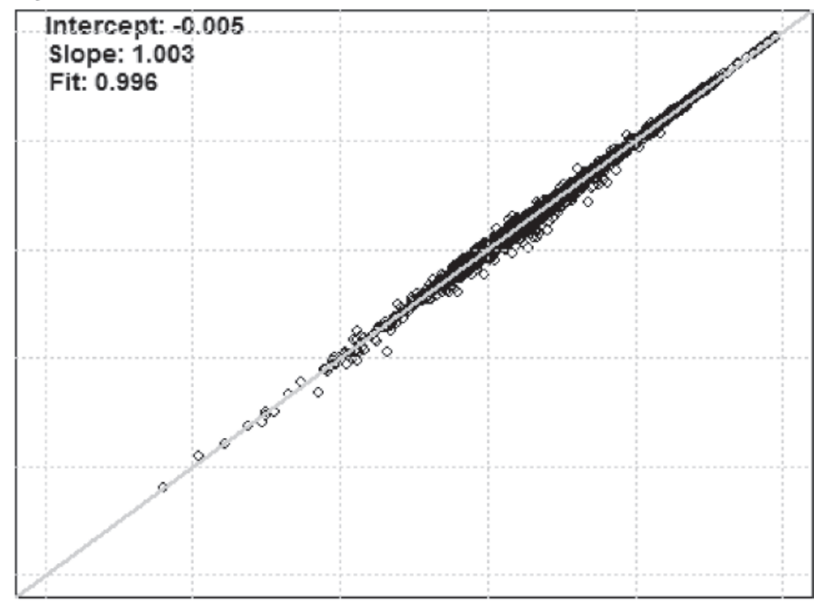

d)

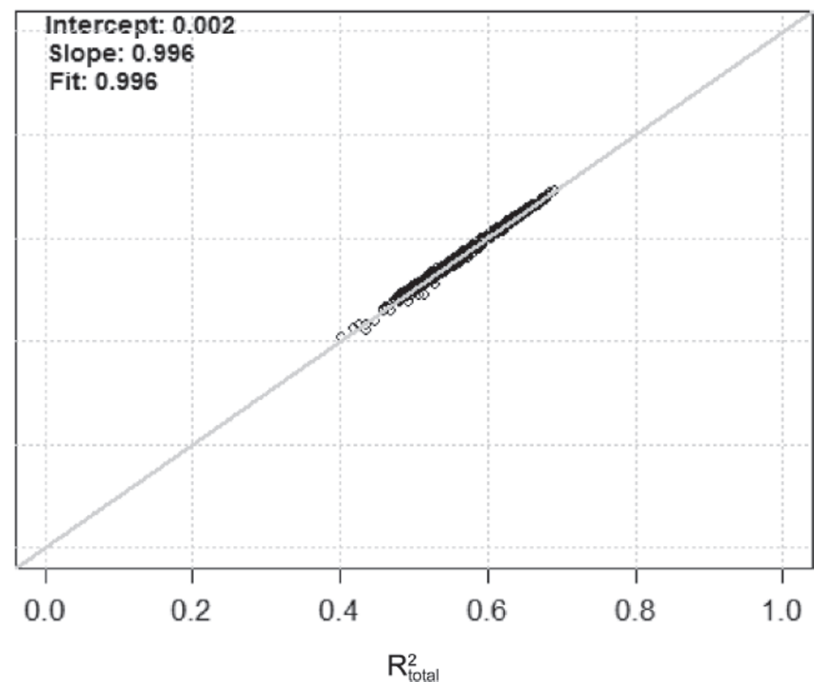

Figure 3. Analytical reliability for single-step $\left(\mathbf{R}_{\text {conv }}^{2}\right)$. derived by direct inversion versus approximated reliability by reduction of contributing nongenotyped (ngt) animals plus diagonalization $\left(\mathbf{R}_{\text {true }}^{2}\right)$ Subplots are for (a) all animals in the pedigree, (b) ngt parents of genotyped (gt) animals only, (c) gt animals only, and (d) gt animals without own or offspring performance. 
animals, including the 6,701 genotyped animals, thus reducing the contributing ngt animals by approximately $90 \%$ (6,695 out of 54,068$)$. The vector $\mathbf{R}_{i m p}^{2}$ used for the approximation using the diagonal matrix approach was calculated explicitly by dividing the diagonal of $\mathbf{A}_{12} \mathbf{A}_{22}^{-1} \mathbf{A}_{21}$ by the diagonal of $\mathbf{A}_{11}$. Finally, when transferring additional information of genomic origin to ngt animals, improved reliabilities for ngt animals were again derived by direct inversion of the left-hand-side of the MME. This means that for this small example, and in contrast to the description of the workflow presented above, all analytical reliabilities involved, except for the approximations tested, were calculated exactly, thus allowing for an evaluation of their efficiency unaffected by the effect of additional approximations that were not the focus of this study.

We present here results based on using both approximations in combination, because this would be most likely the approach when dealing with larger problems in dairy cattle. Figures 2 and 3 summarize our findings. For the example population, the combination of both approximations and the approach proposed to propagate the information of gt animals to ngt animals performed well for all groups of animals investigated. To use this method in the context of large routine evaluations, several fundamental steps of reduction and aggregation of information have to be performed. In this respect, the calculation of single-step reliabilities bears some resemblance to the approach proposed by the Interbull Reliability Task Force (Liu et al., 2017) as a harmonized way of calculating genomic reliabilities. When comparing methodological aspects, the most fundamental difference is the use of imputed genotypes to calculate the PEV of marker effects and therefore to fully take into account the nature of the underlying single-step model in our approach. In contrast, using observed genotypes only, the Interbull method represents a more general approach that might best be labeled "2-step with propagation." The difference in final reliabilities between these 2 approaches might be small, however, especially when applied to small examples developed with the intention of deriving exact values for single-step reliabilities by direct inversion as a reference for comparisons. This does not preclude that in larger applications, depending on the structure of the population and the genotyping coverage, relevant differences might occur. In conclusion, reliabilities of single-step breeding values can be well approximated using the approach presented here. The combination of both approximations presented allows the calculation of analytical reliabilities of high quality, even for larger populations of dairy cattle.

\section{ACKNOWLEDGMENTS}

We gratefully acknowledge the Arbeitsgemeinschaft Süddeutscher Rinderzucht- und Besamungsorganisationen e.V. (Grub, Germany) for their financial support within the research cooperation "Zukunftswege" and the Förderverein Bioökonomieforschung (Bonn, Germany) for their support in the continuous improvement of genomic routine applications. We thank two anonymous reviewers for useful comments and suggestions.

\section{REFERENCES}

Aguilar, I., I. Misztal, D. L. Johnson, A. Legarra, S. Tsuruta, and T. J. Lawlor. 2010. Hot topic: A unified approach to utilize phenotypic, full pedigree,and genomic information for genetic evaluation of Holstein final score. J. Dairy Sci. 93:743-752.

Christensen, O. F., and M. S. Lund. 2010. Genomic prediction when some animals are not genotyped. Genet. Sel. Evol. 42:2.

Fernando, R. L., J. C. M. Dekkers, and D. J. Garrick. 2014. A class of Bayesian methods to combine large numbers of genotyped and non-genotyped animals for whole-genome analyses. Genet. Sel. Evol. 46:50.

Gengler, N., P. Mayeres, and M. Szydlowski. 2007. A simple method to approximate gene content in large pedigree populations: application to the myostatin gene in dual-purpose Belgian Blue cattle. Animal 1:21-28.

Harris, B. L., and D. L. Johnson. 2010. Genomic predictions for New Zealand dairy bulls and integration with national genetic evaluation. J. Dairy Sci. 93:1243-1252.

Liu, Z., P. M. VanRaden, M. H. Lidauer, M. P. Calus, H. Benhajali, H. Jorjani, and V. Ducrocq. 2017. Approximating genomic reliabilities for national genomic evaluation. Interbull Bull. 51:75-85.

Meuwissen, T. H. E., T. Luan, and J. A. Woolliams. 2011. The unified approach to the use of genomic and pedigree information in genomic evaluations revisited. J. Anim. Breed. Genet. 128:429-439.

Shabalina, T., E. C. G. Pimentel, C. Edel, L. Plieschke, R. Emmerling, and K.-U. Goetz. 2017. The role of genotypes from animals without phenotypes in single-step genomic evaluations. J. Dairy Sci. 100:8277-8281. 